\title{
Ancho de distribución eritrocitaria como marcador asociado a riesgo de mortalidad en niños en cuidados intensivos
}

\author{
Red cell distribution width as a marker associated \\ with mortality risk in children in intensive care
}

\author{
María Concepción Rocha-Arrieta', Francisco De la Hoz-Bequis², \\ Ángel Guzmán-Corena ${ }^{3}$, César Muñoz-Mejía ${ }^{4}$, Ángel Castro-Dager ${ }^{5}$
}

Resumen. Introducción. El ancho de distribución eritrocitaria (ADE) ha surgido recientemente como un biomarcador pronóstico de mortalidad y de otros resultados del paciente adulto crítico, pero en niños hay pocos reportes. El objetivo de este estudio fue evaluar la asociación entre el ADE y el riesgo de mortalidad en niños que ingresan a una unidad de cuidados intensivos pediátricos (UCIP). Metodología. Estudio de cohorte prospectivo con 266 pacientes que cumplieron con los criterios de inclusión entre enero y septiembre de 2018. Para el análisis estadístico se utilizó regresión logística multivariada para evaluar la asociación del ADE del primer día y la mortalidad. Se comparó el área bajo la curva ROC del ADE y del Índice Pediátrico de Mortalidad 2 (PIM2). Resultados. Se encontró que un ADE al ingreso mayor de $16,4 \%$ aumentaba la probabilidad de morir, con un OR de $2,6$ (IC95\% 1,17-5,9; $p=0,019)$. La capacidad del ADE para discriminar mortalidad fue moderada (ROC 0,68; IC95\% 0,59-0,76), menor que la del PIM2 (ROC 0,8; IC95\% 0,73-0,86). El ADE y el PIM2 se correlacionaron de manera significativa, aunque débilmente $(r=0,186 ; p<0,002)$. La correlación entre $A D E$ y los días libres de ventilación mecánica fue débil pero significativa $(r=-0,23 ; p<0,001)$. El ADE no se relacionó con los días de uso de medicamentos vasoactivos $(r=0,042 ; p=0,63)$ $n i$ con los días de estancia en UCIP $(r=0,11 ; p=0,07)$. Conclusión. El ADE al ingreso se asoció con un riesgo moderado de mortalidad durante la estancia en UCIP. A pesar de que no demostró ser mejor que el PIM2 para pronosticar mortalidad, por

\footnotetext{
1 Médica, Especialista en Pediatría, Facultad de Medicina, Universidad de Cartagena. Cartagena, Colombia. E-mail: rochamariac201@gmail.com.

${ }^{2}$ Médico, Especialista en Cardiología Pediátrica. Docente del Programa de Pediatría, Facultad de Medicina, Universidad de Cartagena. Cartagena, Colombia.

${ }^{3}$ Médico, Especialista en Pediatría y Cuidados Intensivos. Jefe de la Unidad de Cuidados Intensivos Pediátricos (UCIP) Doña Pilar, Cartagena. Docente del Programa de Pediatría, Facultad de Medicina, Universidad de Cartagena. Cartagena, Colombia.

${ }^{4}$ Médico, Especialista en Pediatría, MSc en Inmunología, Hospital Infantil Napoleón Franco Pareja. Cartagena, Colombia.

${ }^{5}$ Médico, Especialista en Oncohematología Pediátrica. Jefe del Servicio de Oncohematología, Clínica Blas de Lezo, Cartagena. Docente del Programa de Pediatría, Facultad de Medicina, Universidad de Cartagena. Cartagena, Colombia.

Conflicto de interés: los autores declaran que no tienen conflicto de interés.

Medicina \& Laboratorio 2021;25:633-647. https://doi.org/10.36384/01232576.454.

Recibido el 22 de abril de 2021; aceptado el 6 de junio de 2021. Editora Médica Colombiana S.A., 2021 ${ }^{\circ}$.
} 
ser un biomarcador asequible y de bajo costo, podría usarse en conjunto con PIM2 o con otros biomarcadores, con el fin de aumentar su capacidad predictiva en la mortalidad de los niños en cuidados intensivos. Se requieren más estudios que evalúen esta posibilidad en nuestro medio.

Palabras clave: amplitud de distribución eritrocitaria, cuidado crítico, biomarcadores, mortalidad.

Abstract. Introduction. Red cell distribution width (RDW) has recently emerged as a prognostic biomarker for mortality and other outcomes in critically ill adult patients, but there are few reports in children. The aim of this study was to evaluate the association between RDW and the risk of mortality in children admitted to a pediatric intensive care unit (PICU). Methodology. Prospective cohort study with 266 patients who met the inclusion criteria between January and September 2018. For the statistical analysis, multivariate logistic regression was used to evaluate the association of RDW on the first day and mortality. The areas under the ROC curve for $A D E$ and the Pediatric Mortality Index 2 (PIM2) were compared. Results. A RDW at admission greater than $16.4 \%$ increased the probability of death, with an OR of 2.6 (95\% Cl 1.17-5.9; $p=0.019)$. The ability of $A D E$ to discriminate mortality was moderate $(R O C) 0.68 ; 95 \% \mathrm{Cl} 0.59-0.76)$, lower than that of the PIM2 (ROC 0.8; $95 \% \mathrm{Cl}$ 0.73-0.86). ADE and PIM2 were significantly correlated, albeit weakly ( $r=0.186$; $p<0.002)$. The correlation between RDW and mechanical ventilation-free days was weak but significant $(r=-0.23 ; p<0.001)$. The $R D W$ was not related to the days of use of vasoactive drugs $(r=0.042 ; p=0.63)$ or to the days of stay in the PICU ( $r=0.11$; $p=0.07)$. Conclusion. $R D W$ on admission was associated with a moderate risk of mortality during the stay in the PICU. Although it did not prove to be better than PIM2 in predicting mortality, as it is an affordable and low-cost biomarker, it could be used in conjunction with PIM2 or with other biomarkers, in order to increase its predictive ability for mortality in critically ill children. More studies are required to evaluate this possibility in our setting.

Keywords: red blood distribution width, critical care, biomarkers, mortality.

\section{Introducción}

Las Unidades de Cuidados Intensivos Pediátricos (UCIP) se han constituido en una herramienta esencial para la atención de los niños con enfermedades graves en los últimos 50 años [1,2]. La mortalidad en las unidades se ha reducido de un $16 \%$ hasta un $2,5 \%$ a $5 \%$ en países desarrollados [2,3]. En América Latina es un poco más alta $(8,9 \%)$, y en Colombia es mucho mayor (de 6,1\% hasta $31,8 \%$ ) $[4,5]$. Las escalas de seve- ridad se han implementado en las UCIP para clasificar la condición del paciente al momento del ingreso y predecir la mortalidad, además, permiten realizar comparaciones inter e intra-unidad a nivel local, nacional e internacional [6].

El Índice Pediátrico de Mortalidad 2 (PIM2) ha demostrado tener un adecuado valor predictivo, se ajusta a diferentes grupos de riesgo y ha sido validado en países desarrollados y en vías de desarrollo $[4,7,8]$. Sin embargo, los punta- 
jes predictivos de la mortalidad no han sido creados para tomar decisiones individuales a nivel clínico ni para seleccionar pacientes para ensayos clínicos [9]. Adicionalmente, se ha observado que tienen un menor desempeño cuando se evalúan bajo algunas condiciones como la sepsis [10,11]; afortunadamente, algunos biomarcadores han mostrado potencial para compensar estas limitaciones [10-12], como sucede con la combinación de interleuquina 6 (IL-6), pétido natriurético tipo $B$ (BNP) e INR en la predicción de la mortalidad a 28 días por sepsis [13].

El ancho de distribución eritrocitaria (ADE) es una medida de la variabilidad del tamaño de los glóbulos rojos, disponible en todos los analizadores de hematología automatizados [14]. Aunque inicialmente la utilidad clínica del ADE se limitó al diagnóstico diferencial de la anemia [15], ha surgido como un biomarcador prometedor en la predicción de mortalidad en el cuidado crítico en adultos con falla cardíaca, accidente cerebrovascular, neumonía, sepsis, y en general, en el paciente en $\mathrm{UCl}$ [16-19]. El impacto biológico de las enfermedades graves con variabilidad del ADE continúa en estudio, especialmente en el contexto de sepsis grave y shock séptico, donde las mediciones seriadas del ADE podrían utilizarse como factor pronóstico [20-24]. Así mismo, se viene investigando en COVID-19, donde podría utilizarse como un biomarcador simple y confiable para predecir la gravedad y clasificar el riesgo [25-30], y en pacientes con neumonía asociada a ventilador, donde junto al índice neutrófilo-linfocito ha demostrado correlación con mortalidad [31].

En pediatría, en particular, el interés por estudiar la asociación del ADE con mortalidad ha venido en aumento [3237]. Los primeros estudios realizados fueron durante el posquirúrgico de cardiopatías congénitas complejas, y se ha ampliado su uso en cuidado intensivo en general. En estudios recientes se ha demostrado la utilidad del ADE [36-38], y podría ser un biomarcador independiente de magnitud suficiente para influir en el resultado de una enfermedad crítica [34-37,39,40], e inclusive como predictor del uso de ventilación mecánica, ya que la hipoxemia desencadena la liberación de reticulocitos inmaduros a la circulación, lo que aumenta a su vez, el ADE en el paciente afectado $[16,33,36]$.

El objetivo de este trabajo fue evaluar la asociación entre el ADE y el riesgo de mortalidad en niños en una UCIP, en el contexto de un país en vías de desarrollo.

\section{Metodología}

Se realizó un estudio de cohorte prospectivo, entre enero y septiembre de 2018, en la Unidad de Cuidados Intensivos Pediátricos "Doña Pilar", ubicada en la ciudad de Cartagena de Indias, en la costa norte colombiana. La presente investigación es un estudio Tipo A, el cual, de acuerdo a la normatividad colombiana, corresponde a investigación de bajo riesgo, pues los datos obtenidos son tomados a partir de las historias clínicas. El estudio fue aprobado por el comité de ética institucional.

\section{Población y recolección de datos}

Se incluyeron niños mayores de un mes de vida y menores de 16 años que ingresaron a la UCIP. Todos los pacientes provenían del mismo centro pediátrico "Hospital Infantil Napoleón Franco Pareja". En total se identificaron 642 
pacientes durante el tiempo de estudio, de estos, 266 individuos fueron analizados (figura 1). Se excluyeron aquellos individuos procedentes de otra UCIP, los que fueron remitidos a otra UCIP, si fueron dados de alta en las primeras 24 horas luego del ingreso, si habían tenido estancia en UCIP en los últimos tres meses, antecedente de alguna hemoglobinopatía congénita, diagnóstico de tumor sólido o neoplasia hematolinfoide, además, que hubiesen recibido trasplante de médula ósea o transfusión de glóbulos rojos en los 14 días previos a su ingreso a cuidados intensivos. Los datos clínicos y de laboratorio fueron obtenidos a partir de las historias clínicas.

\section{Variables de exposición y desenlace}

Se obtuvieron los siguientes datos: 1) demográficos: edad y sexo; 2) clínicos: antecedentes, diagnósticos de ingreso y comorbilidades. Además, se calculó la razón de mortalidad estandarizada (REM) para la cohorte, comparando la mortalidad observada y la predicha por el PIM2, el cual por protocolo institucional es calculado en las primeras horas de ingreso; 3) características de la hospitalización: días libres de ven- tilación mecánica, días de estancia en UCIP y días de uso de medicamentos vasoactivos-inotrópicos.

Como variables de laboratorio se registraron parámetros obtenidos del cuadro hemático de IV generación (Advia Hematology Analyzer, Siemens Healthcare Diagnostics, Deerfield, IL, USA); el ADE tiene como valor de referencia institucional $11,5 \%$ a $14,5 \%$. Además, se documentaron otras variables hematológicas (hemoglobina, hematocrito y volumen corpuscular medio). La albúmina sérica fue también medida en algún punto durante la estancia en UCIP. A todos los pacientes ingresados a la UCIP se les toma muestra para hemograma que se procesa durante las primeras 24 horas de estancia, como parte del protocolo institucional.

El desenlace principal analizado fue mortalidad; de manera secundaria se evaluó la relación del ADE con la necesidad de ventilación mecánica, días libres de ventilación mecánica (días sin ventilación mecánica en UCIP/días de hospitalización totales en UCIP), necesidad y días de utilización de vasoactivos-inotrópicos y días de estancia en UCIP.

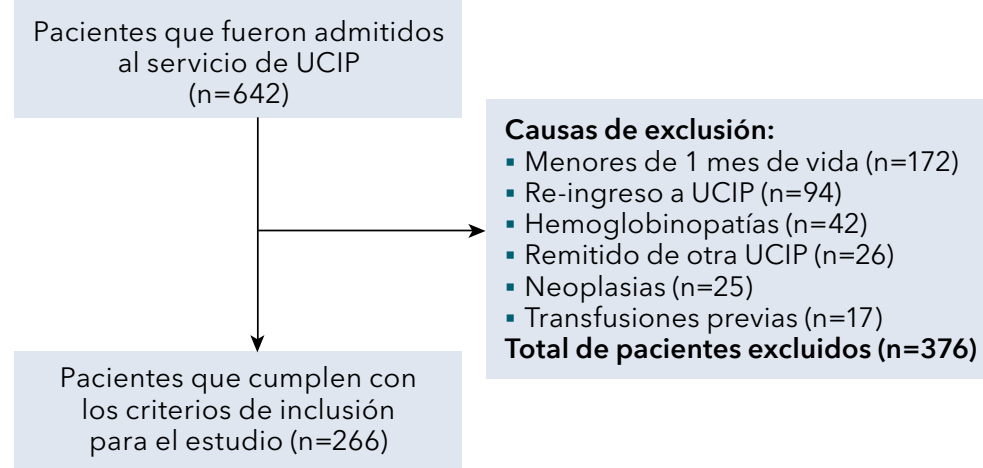

Figura 1. Flujograma de selección de los individuos que participaron en el estudio. 


\section{Análisis estadístico}

Todas las variables evaluadas son presentadas mediante medidas de tendencia central y de dispersión para los datos continuos, y para los categóricos se usaron frecuencias absolutas y relativas.

El contraste de hipótesis en el estudio de grupos (niños que fallecen versus quienes no fallecen), se realizó mediante las pruebas de t de student y de análisis de varianza (ANOVA) para variables continuas; $X^{2}$ de independencia o de Fisher para variables categóricas.

La variable continua de ADE fue convertida en una variable categórica. Esto se realizó de acuerdo a su distribución según las medidas de posición, usando específicamente los valores de ADE inferiores al primer cuartil o superiores al tercer cuartil, luego se realizó una comparación intercuartil, como lo registran informes previos [1,36,37].

La asociación de otras variables demográficas, clínicas y de laboratorio con el desenlace de la mortalidad, fue estimada mediante regresión logística no condicional, que permitió calcular las OR con sus respectivos IC95\% ajustados. Las covariables de los modelos de regresión logística multivariado fueron escogidas de acuerdo a la plausibilidad biológica, disponibilidad de los datos y estudios previos $[1,36,41]$. Un valor de $p<0,05$ fue considerado como estadísticamente significativo.

El área bajo la curva ROC se utilizó para determinar el punto de corte del ADE con mejor sensibilidad, especificidad y valores predictivos positivos y negativos para predecir mortalidad. La relación entre el ADE y la escala de PIM2 se valoró a través del índice de correlación de Pearson. También se determinó el área bajo la curva ROC para el PIM2, y se comparó con la del ADE mediante la prueba de Hanley y McNeil.

La correlación entre el ADE y los desenlaces secundarios (días libres de ventilación mecánica, días de estancia en UCIP y días de uso de medicamentos vasoactivos-inotrópicos) fue evaluada mediante el índice de correlación de Pearson. Los datos y gráficas fueron obtenidos utilizando el paquete de análisis estadístico SPSS v.20.

\section{Resultados}

En total se identificaron 642 pacientes, de estos, 266 individuos fueron incluidos en el análisis. El 53,4\% fueron hombres y la edad media fue de 21,5 meses $(6,75-109,25)$.

Como antecedentes importantes, el $18 \%$ de los todos los individuos habían estado en UCIP previamente, el 11\% estuvieron en ventilación mecánica durante la etapa neonatal y el mismo porcentaje también lo estuvo posteriormente. La desnutrición fue el principal antecedente patológico en el $21 \%$ de la población, seguido del cardíaco $(17,3 \%)$, neurológico $(13,5 \%)$ y respiratorio $(11,7 \%)$.

En promedio, la enfermedad que motivó el ingreso a UCIP tuvo una duración de tres días. Las principales etiologías fueron de origen respiratorio $(65,4 \%)$, infeccioso (37,2\%), neurológico $(22,6 \%)$ y cardiovascular (18,4\%). El PIM2 general tuvo una mediana de $2,8(1,1-7,12)$. Respecto a las variables hematológicas, el $49,6 \%$ de los niños tenían anemia. En el $69,5 \%$ de los individuos se logró determinar la albúmina, encontrándose disminuida en la gran mayoría.

El $60 \%$ de los individuos estuvieron en ventilación mecánica durante la estan- 
cia en UCIP, con una mediana de 4 días. El $47 \%$ necesitaron medicamentos vasoactivos, con una mediana de 5 días. La mediana de días de estancia en UCIP fue de 7. La mortalidad esperada fue de 7,1\%; sin embargo, la mortalidad observada fue del $17,3 \%$ en esta población.

\section{Distribución del ADE}

Los puntos de corte correspondientes a los percentiles 25, 50 y 75 del ADE fueron $13,5 \%, 14,7 \%$ y $16,4 \%$, respectivamente. Las comparaciones de las distintas variables evaluadas de acuerdo a su distribución en el cuartil inferior y cuartil superior se encuentran en la tabla 1.

El número de días de la enfermedad actual, la proporción de desnutrición, anemia, sepsis, shock al ingreso y a las 24 horas, ventilación mecánica, uso de vasoactivos y la mortalidad fueron significativamente mayores en el grupo del cuartil superior del ADE comparado con el cuartil inferior (tabla 1). Las variables hematológicas como hemoglobina, hematocrito y volumen corpuscular medio fueron significativamente menores en el cuartil superior del ADE, como se esperaba.

\section{Variables relacionadas con mortalidad}

En la asociación cruda, las variables que se relacionaron con la mortalidad fueron: un $A D E$ en el cuartil superior de su distribución, el antecedente de ventilación mecánica durante la etapa neonatal o en cualquier otra etapa de la vida previo al ingreso, la desnutrición, la presencia de sepsis, enfermedad neurológica, enfermedad gastrohepática, shock al ingreso, shock en las primeras 24 horas posterior al ingreso, un mayor número de días de enfer- medad, un PIM2 más alto, la anemia al ingreso y una menor albúmina sérica (tabla 2). Después de ajustar por las variables significativas y biológicamente plausibles, se mantuvieron asociadas significativamente con la mortalidad, las siguientes variables: el cuartil superior del ADE [OR 2,64 (IC95\% 1,17$5,9 ; p=0,019)]$, el ADE [OR 1,21 (IC95\% $1,0-1,45 ; p=0,042)]$, el haber estado en UCIP previamente [OR 2,7 (IC95\% 1,1$7,0 ; p=0,031)]$, el shock 24 horas posterior al ingreso [OR 14,3 (IC95\% 5,239; $p<0,001)$ ], el PIM2 [OR 1,07 (IC95\% $1,03-1,12 ; p<0,001)]$, la albúmina [OR 0,34 (IC95\% 0,18-0,65; $p<0,001)$ ], el uso de ventilación mecánica [OR 25,3 (IC95\% 3,2-199,7; $p<0,002$ )], los días en ventilación mecánica [OR 1,05 (IC95\% 1,00-1,11; $p=0,032$ )], y el uso de vasoactivos [OR 65 (IC95\% 8-11; $p<0,001)$ ] (tabla 2).

\section{Relación entre el ADE y mortalidad}

Un ADE mayor a 16,4\% aumentó la posibilidad de morir 2,64 veces (IC95\% $1,17-5,9 ; p=0,019)$. Al evaluar la relación de este posible marcador con la escala de mortalidad PIM2, se correlacionaron de manera significativa, aunque débilmente $(r=0,186 ; p<0,002)$. La REM para toda la cohorte fue de 2,43, siendo en el cuartil inferior 1,8 , mientras que aumentó a 2,9 en cuartil superior.

El ADE mostró un ROC moderado para discriminar mortalidad (ROC 0,68; IC95\% 0,59-0,76) (figura 2). El punto de corte del ADE escogido para mortalidad fue mayor o igual a $15,5 \%$, teniendo en cuenta que se prefiere una prueba con la menor tasa de falsos negativos, sin disminuir la especificidad. Este valor tenía una sensibilidad del $65,2 \%$, una especificidad del $68,2 \%$, un valor predictivo positivo del $30 \%$ y un valor predictivo negativo del $90 \%$. 
Tabla 1. Características demográficas, clínicas y de laboratorio de la población

\begin{tabular}{lcccc}
\hline Características & $\begin{array}{c}\text { Total } \\
(\mathbf{n = 2 6 6 )}\end{array}$ & $\begin{array}{c}\text { Cuartil inferior } \\
\text { ADE } \\
(\mathbf{n = 6 6 )}\end{array}$ & $\begin{array}{c}\text { Cuartil superior } \\
\text { ADE } \\
(\mathbf{n = 6 6 )}\end{array}$ & Valor $\mathbf{p}^{*}$ \\
\hline Edad (meses) & 21,5 & $4(1,9-108,6)$ & $9(4,9-93,7)$ & 0,61 \\
\hline Antecedentes & $(6,75-109,25)$ & & $18(28,3 \%)$ & 0,14 \\
\hline Estancia en UCIN previa & $52(19,5 \%)$ & $11(16,7 \%)$ & $13(19,7 \%)$ & 0,074 \\
\hline $\begin{array}{l}\text { Pretérmino } \\
\text { VM durante etapa neonatal }\end{array}$ & $29(10,9 \%)$ & $5(7,6 \%)$ & $10(15,2 \%)$ & 0,76 \\
\hline $\begin{array}{l}\text { VM previamente } \\
\text { Estancia en UCIP }\end{array}$ & $29(10,9 \%)$ & $3(4,5 \%)$ & $11(16,7 \%)$ & 0,18 \\
previamente & $48(18 \%)$ & $11(16,7 \%)$ & $15(22,7 \%)$ & 0,38
\end{tabular}

\section{Comorbilidades al \\ ingreso}

Respiratoria

$\begin{array}{cccc}31(11,7 \%) & 7(10,6 \%) & 8(12,1 \%) & 0,78 \\ 46(17,3 \%) & 5(7,6 \%) & 12(18,2 \%) & 0,11 \\ 5(1,9 \%) & 2(3 \%) & 2(3 \%) & 0,999 \\ 36(13,5 \%) & 8(12,1 \%) & 6(9,1 \%) & 0,77 \\ 19(7,1 \%) & 3(4,5 \%) & 8(12,1 \%) & 0,2 \\ 56(21,1 \%) & 7(10,6 \%) & 23(34,8 \%) & 0,002^{\star} \\ 7(2,6 \%) & 2(3 \%) & 1(1,5 \%) & 0,999\end{array}$

\section{Morbilidad en UCI}

\begin{tabular}{lcccc} 
Días de enfermedad actual & $3(1-7)$ & $3(2-6)$ & $5(2-5)$ & $0,025^{*}$ \\
Sepsis & $99(37,2 \%)$ & $22(33,3 \%)$ & $35(53 \%)$ & $0,022^{*}$ \\
Shock & $76(28,6 \%)$ & $13(19,7 \%)$ & $25(37,9 \%)$ & $0,021^{*}$ \\
Shock 24 h posingreso & $81(30 \%)$ & $12(18,2 \%)$ & $32(48,5 \%)$ & $<0,001^{*}$ \\
Respiratoria & $174(65,4 \%)$ & $44(66,7 \%)$ & $50(75,8 \%)$ & 0,24 \\
Cardiovascular & $49(18,4 \%)$ & $7(10,6 \%)$ & $15(22,7 \%)$ & 0,06 \\
Neurológica & $60(22,6 \%)$ & $17(25,8 \%)$ & $17(25,8 \%)$ & 0,999 \\
Hematológica & $4(1,5 \%)$ & $1(1,5 \%)$ & $2(3 \%)$ & 0,999 \\
Gastrointestinal & $20(7,5 \%)$ & $4(6,1 \%)$ & $4(6,1 \%)$ & 0,999 \\
Renal & $26(9,8 \%)$ & $4(6,1 \%)$ & $6(9,1 \%)$ & 0,74 \\
Trauma & $6(2,3 \%)$ & $1(1,5 \%)$ & 0 & 0,999 \\
Endocrinológica & $10(3,8 \%)$ & $5(7,6 \%)$ & $2(3 \%)$ & 0,44 \\
\hline
\end{tabular}




\begin{tabular}{|c|c|c|c|c|}
\hline POP CV sin bomba & $2(0,8 \%)$ & $1(1,5 \%)$ & $1(1,5 \%)$ & 0,999 \\
\hline POP CV con bomba & $24(9 \%)$ & $1(1,5 \%)$ & $6(9,1 \%)$ & 0,11 \\
\hline $\begin{array}{l}\text { Posquirúrgico diferente } \\
\text { a CV }\end{array}$ & $6(2,3 \%)$ & 0 & $1(1,5 \%)$ & 0,999 \\
\hline PIM2 & $2,8(1,1-7,12)$ & $5,5(2,2-16)$ & $8,4(4,3-19,1)$ & $0,007^{*}$ \\
\hline REM & $2,43(1,7-3,2)$ & $1,8(0,74-3,7)$ & $2,9(1,9-4,5)$ & \\
\hline \multicolumn{5}{|l|}{ Datos hematológicos } \\
\hline ADE (\%) & $14,7(13,58-16,4)$ & $13,1(12,46-13,37)$ & $17,6(16,9-18,1)$ & $<0,001$ * \\
\hline Anemia para la edad & $132(49,6 \%)$ & $26(39,4 \%)$ & $47(71,2 \%)$ & $<0,001^{*}$ \\
\hline Hemoglobina (g/dL) & $10,4(8,9-12,3)$ & $10,4(9,2-12,1)$ & $9,4(8,3-11)$ & $<0,001$ * \\
\hline Hematocrito (\%) & $31,9(27,1-36,6)$ & $31,1(27,1-35,1)$ & $29,3(26-34,1)$ & $<0,001^{*}$ \\
\hline $\begin{array}{l}\text { Volumen corpuscular } \\
\text { medio (VCM) (fL) }\end{array}$ & $80(74,1-84)$ & $81,6(77,9-85,2)$ & $74(66-82)$ & $<0,001^{*}$ \\
\hline Albúmina $(\mathrm{g} / \mathrm{dL})(\mathrm{n}=185)$ & $2,9(2,27-3,5)$ & $\begin{array}{c}2,9(2,4-3,4) \\
n=40\end{array}$ & $\begin{array}{c}2,9(2,2-3,4) \\
n=53\end{array}$ & 0,67 \\
\hline \multicolumn{5}{|l|}{ Desenlaces } \\
\hline Uso de VM & $158(59,4 \%)$ & $30(45,5 \%)$ & $48(72,7 \%)$ & 0,001 * \\
\hline Días de VM (n=158) & $4(2-8)$ & $6(2,7-12,5)$ & $5(3-8,5)$ & 0,86 \\
\hline VM prolongada (7 días) & $46(17,2 \%)$ & $13(48,1 \%)$ & $14(51,9 \%)$ & 0,82 \\
\hline Uso de vasoactivos & $126(47,4 \%)$ & $23(34,8 \%)$ & $38(57,6 \%)$ & $0,009 *$ \\
\hline $\begin{array}{l}\text { Días con vasoactivos } \\
(n=126)\end{array}$ & $5(3-10)$ & $6(2,7-14,2)$ & $5,5(3-12,7)$ & 0,77 \\
\hline Mortalidad & $46(17,3 \%)$ & $7(10,6 \%)$ & $22(33,3 \%)$ & $0,002^{*}$ \\
\hline Días de estancia en UCIP & $7(4-12)$ & $11(7,7-19,5)$ & $11,5(4-18,2)$ & 0,27 \\
\hline
\end{tabular}

${ }^{*} p<0,05$.

La relación del ADE con las variables cuantitativas independientes se presenta como mediana (RIC) y con las variables cualitativas con $n(\%)$.

ADE: ancho de distribución eritrocitaria; VM: ventilación mecánica; POP CV: posoperatorio cardiovascular; REM: razón de mortalidad estandarizada; UCIP: unidad de cuidados intensivos pediátricos; UCIN: unidad de cuidados intensivos neonatales; PIM2: índice pediátrico de mortalidad 2.

Tabla 2. Análisis multivariado de asociación con mortalidad

\begin{tabular}{lccccc}
\hline Mortalidad & Sí (n=46) & No (n=226) & Valor $\boldsymbol{p}^{*}$ & $\begin{array}{c}\text { OR crudo } \\
\text { IC95\% } \boldsymbol{p}\end{array}$ & $\begin{array}{c}\text { OR ajustado* } \\
\text { IC95\% } \boldsymbol{p}\end{array}$ \\
\hline Variables & \multirow{2}{*}{$\begin{array}{l}\text { ICsnutrición } \\
\text { 17(37\%) }\end{array}$} & $39(17,7 \%)$ & 0,004 & $\begin{array}{c}2,72(1,36-5,43) \\
0,005^{*}\end{array}$ & $\begin{array}{c}1,84(0,8-4,2) \\
0,15\end{array}$ \\
\hline
\end{tabular}


Ancho de distribución eritrocitaria como marcador asociado a riesgo de mortalidad en niños en cuidados intensivos

\begin{tabular}{|c|c|c|c|c|c|}
\hline $\begin{array}{l}\text { Intubación } \\
\text { previa }\end{array}$ & $10(21,7 \%)$ & $19(8,6 \%)$ & 0,01 & $\begin{array}{c}2,9(1,26-6,83) \\
0,012^{\star}\end{array}$ & $\begin{array}{c}2,24(0,77-6,5) \\
0,13\end{array}$ \\
\hline $\begin{array}{l}\text { Estancia en } \\
\text { UCIP previa }\end{array}$ & $14(30,4 \%)$ & $34(15,5 \%)$ & 0,016 & $\begin{array}{c}2,39(1,15-4,9) \\
0,019^{\star}\end{array}$ & $\begin{array}{c}2,7(1,1-7,0) \\
0,031 *\end{array}$ \\
\hline Ingreso sepsis & $28(60,9 \%)$ & $71(32,3 \%)$ & $<0,001$ & $\begin{array}{c}3,2(1,69-6,2) \\
<0,001^{\star}\end{array}$ & $\begin{array}{c}1,75(0,76-4,02) \\
0,18\end{array}$ \\
\hline $\begin{array}{l}\text { Ingreso } \\
\text { neurológico }\end{array}$ & $17(37 \%)$ & $43(19,5 \%)$ & 0,01 & $\begin{array}{c}2,4(1,2-4,7) \\
0,012^{\star}\end{array}$ & $\begin{array}{c}1,62(0,7-3,7) \\
0,25\end{array}$ \\
\hline $\begin{array}{l}\text { Ingreso } \\
\text { gastrointestinal }\end{array}$ & $8(17,4 \%)$ & $12(5,5 \%)$ & 0,005 & $\begin{array}{c}3,64(1,39-9,5) \\
0,00{ }^{\star}\end{array}$ & $\begin{array}{c}2,6(0,8-9,0) \\
0,11\end{array}$ \\
\hline $\begin{array}{l}\text { Días de } \\
\text { enfermedad }\end{array}$ & $5(2,7-8)$ & $3(1-6,7)$ & 0,002 & $\begin{array}{l}1,04(1,004- \\
1,080) 0,03^{*}\end{array}$ & $\begin{array}{c}1,01(0,97-1,06) \\
0,45\end{array}$ \\
\hline $\begin{array}{l}\text { Shock al } \\
\text { ingreso }\end{array}$ & $26(56,5 \%)$ & $50(22,7 \%)$ & $<0,001$ & $\begin{array}{c}4,4(2,2-8,5) \\
<0,001^{*}\end{array}$ & $\begin{array}{c}1,5(0,6-3,7) \\
0,3\end{array}$ \\
\hline $\begin{array}{l}\text { Shock } 24 \text { h } \\
\text { posingreso }\end{array}$ & $38(82,6 \%)$ & $43(19,5 \%)$ & $<0,001$ & $\begin{array}{c}19,5(8,5-44,9) \\
<0,001^{\star}\end{array}$ & $\begin{array}{c}14,3(5,2-39) \\
<0,001^{*}\end{array}$ \\
\hline PIM2 & $8,6(4,5-25)$ & $2,1(1-5,75)$ & $<0,001$ & $\begin{array}{c}1,09(1,05-1,13) \\
<0,001^{*}\end{array}$ & $\begin{array}{c}1,07(1,03-1,12) \\
<0,001^{*}\end{array}$ \\
\hline Anemia & $29(63 \%)$ & $103(46,8 \%)$ & 0,045 & $\begin{array}{l}1,93(1,007- \\
3,72) 0,048^{\star}\end{array}$ & $\begin{array}{c}1,5(0,46-5,07) \\
0,48\end{array}$ \\
\hline Hemoglobina & $\begin{array}{c}9,74 \\
(8,3-11,2)\end{array}$ & $10,8(9-12,4)$ & 0,027 & $\begin{array}{c}0,85(0,73-0,99) \\
0,036^{\star}\end{array}$ & $\begin{array}{c}0,92(0,76-1,11) \\
0,401\end{array}$ \\
\hline $\begin{array}{l}\text { Albúmina } \\
(n=185)\end{array}$ & $2,3(2-2,9)$ & $\begin{array}{c}3,03 \\
(2,45-3,56)\end{array}$ & $<0,001$ & $\begin{array}{c}0,3(0,17-0,52) \\
<0,001^{*}\end{array}$ & $\begin{array}{c}0,34(0,18-0,65) \\
<0,001^{*}\end{array}$ \\
\hline $\begin{array}{l}\text { VM durante } \\
\text { etapa neonatal }\end{array}$ & $9(19,6 \%)$ & $20(9,1 \%)$ & 0,038 & $\begin{array}{c}2,43(1,02-5,75) \\
0,041^{\star}\end{array}$ & $\begin{array}{c}1,89(0,65-5,4) \\
0,23\end{array}$ \\
\hline Uso de VM & 45 (95,8\%) & $113(51,4 \%)$ & $<0,001$ & $\begin{array}{c}42,6(5,7-314,5) \\
<0,001^{\star}\end{array}$ & $\begin{array}{c}25,3(3,2-199,7) \\
<0,002^{\star}\end{array}$ \\
\hline $\begin{array}{l}\text { Días en VM } \\
(n=158)\end{array}$ & $4(2,5-6)$ & $4(2-6)$ & 0,01 & $\begin{array}{c}1,04(1,004-1,09) \\
0,033^{*}\end{array}$ & $\begin{array}{c}1,05(1,00-1,11) \\
0,032^{*}\end{array}$ \\
\hline $\begin{array}{l}\text { Uso de } \\
\text { vasoactivos }\end{array}$ & 45 (95,8\%) & $81(36,8 \%)$ & $<0,001$ & $\begin{array}{c}77,2(10,4-570) \\
<0,001^{\star}\end{array}$ & $\begin{array}{l}65(8-11) \\
<0,001^{\star}\end{array}$ \\
\hline ADE & $\begin{array}{c}16,3 \\
(14,6-17,6)\end{array}$ & $\begin{array}{c}14,5 \\
(13,5-15,9)\end{array}$ & $<0,001$ & $\begin{array}{c}1,3(1,13-1,5) \\
0,003^{*}\end{array}$ & $\begin{array}{c}1,21(1,0-1,45) \\
0,042^{\star}\end{array}$ \\
\hline $\begin{array}{l}\text { ADE (cuartil } \\
\text { superior) }\end{array}$ & $22(47,8 \%)$ & $44(20 \%)$ & $<0,001$ & $\begin{array}{c}3,66(1,88-7,13) \\
<0,001^{\star}\end{array}$ & $\begin{array}{c}2,64(1,17-5,9) \\
0,019^{\star}\end{array}$ \\
\hline
\end{tabular}

${ }^{*} p<0,05$.

$\mathrm{X}^{2}$ o test de Fisher según corresponda.

\#Modelo de regresión ajustado por edad, género, hemoglobina, desnutrición, sepsis al ingreso, shock al ingreso y el PIM2.

ADE: ancho de distribución eritrocitaria; VM: ventilación mecánica; UCIP: unidad de cuidados intensivos pediátricos; PIM2: índice pediátrico de mortalidad 2. 
Sin embargo, cuando se utilizaron diferentes puntos de corte para identificar los grupos de bajo riesgo versus los de alto riesgo, los individuos con un ADE menor a 13,5\% (límite superior del cuartil inferior) tenían un riesgo de morir del $24,1 \%$, comparados con los sujetos que tenían un $A D E$ mayor a $16,4 \%$, quienes tenían un riesgo de mortalidad del $75,9 \%(p=0,002)$ para ambos desenlaces. El valor predictivo negativo para mortalidad de un ADE $<13,4 \%$ fue del $89,4 \%$ y el valor predictivo positivo del ADE $>16,4 \%$ fue del $33,3 \%$.

El área bajo la curva del PIM2 fue mayor que la del ADE (ROC 0,8; IC95\% $0,73-0,86$ versus ROC 0,68; IC95\% $0,59-0,76)$, respectivamente (figura 3 ).

\section{Relación entre el ADE y desenlaces secundarios}

El análisis de regresión lineal demostró una correlación inversa débil, pero significativa, entre los días libres de ventilación mecánica durante la estancia en UCIP y el ADE $(r=-0,23 ; p=0,001)$. A pesar de que hubo una proporción significativamente mayor de niños ventilados en el grupo del cuartil superior del ADE comparado con el primer cuartil, esta diferencia no se mantuvo en cuanto al número de días de ventilación mecánica y frecuencia de ventilación mecánica prolongada (tabla 1). Por otro lado, el ADE no se correlacionó con los días de uso de medicamentos vasoactivos $(r=0,042 ; p=0,63)$ ni con los días de estancia en UCIP $(r=0,11 ; p=0,07)$.

\section{Discusión}

Este constituye el primer estudio en Colombia que evalúa el desempeño del ADE como biomarcador para la determinación de riesgo de muerte en la UCIP. Varios estudios con objetivos y contexto similar han sido identificados por otros grupos [32-37]. El ADE al ingreso mostró una capacidad moderada para estimar la mortalidad

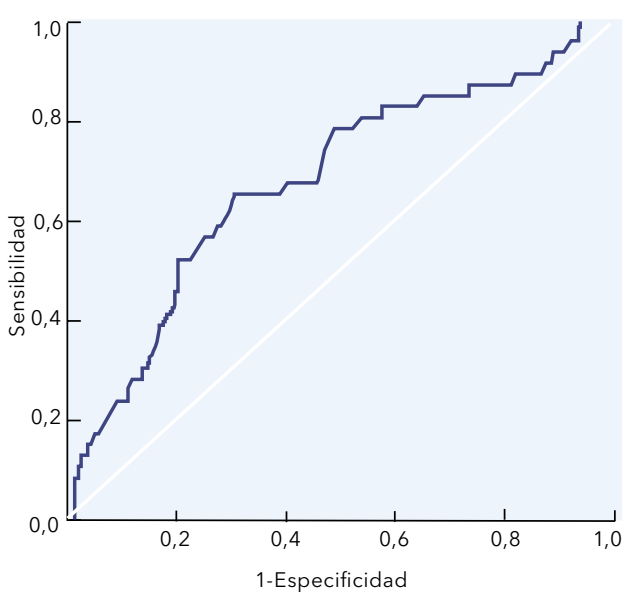

Figura 2. Curva $R O C$ del ancho de distribución eritrocitaria (ADE) para predecir mortalidad.

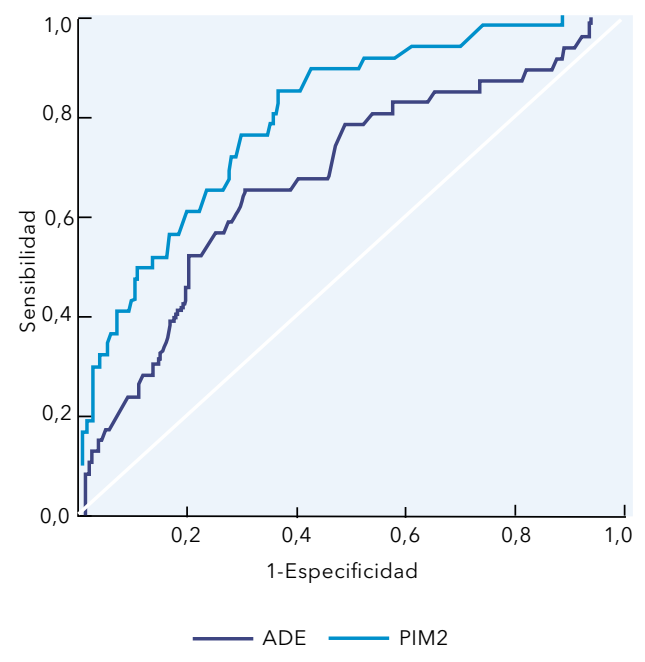

Figura 3. Comparación de las curvas ROC del ancho de distribución eritrocitaria (ADE) y del índice pediátrico de mortalidad 2 (PIM2) para predecir mortalidad. 
en niños que son admitidos a nuestra UCIP, aunque inferior al PIM2. No obstante, se estableció como un indicador independiente en los modelos de asociación. En nuestra población no hubo relación entre la edad de los individuos y la distribución del ADE, contrario a lo reportado por Said y colaboradores, y Ramby y colaboradores, quienes describieron que los niños con menor edad tenían un ADE mayor [36,37]; Parker planteó que esta diferencia puede deberse a una mayor cantidad de hemoglobina fetal en los individuos menores [15], lo que supone la necesidad de medir esta posible variable de confusión en próximos estudios.

Said y colaboradores reportaron de manera similar, que el ADE de ingreso y el cambio relativo en el ADE en los primeros 7 días, se relacionaban con la mortalidad y/o el uso de ECMO (del inglés, Extracorporeal Membrane Oxygenation), luego de ajustar por la gravedad de la enfermedad [36]. Estos resultados son similares a lo reportado en adultos $[1,41]$.

La capacidad operativa del ADE en nuestro estudio fue similar a la reportada por Ramby y colaboradores (ROC 0,65 ; IC95\% 0,55-0,75), y Sadi y colaboradores (ROC 0,611; IC95\% 0,076$0,218)[36,37]$. En este último estudio, el PIM2 mostró un mejor desempeño que el $A D E$, al igual que en nuestra población. Nuestros datos se suman a la evidencia de la sobre discriminación del PIM2 en países de la región, sin embargo, fue mayor el número de muertes observadas que las esperadas, esto hace referencia a las deficiencias de calibración de la prueba que se presentan cuando se aplican a nuestra región, como lo demostró Arias y colaboradores [4].

La desnutrición mostró relación con un ADE en el cuartil superior y con la mor- talidad. La desnutrición podría ser un factor importante en países en vías de desarrollo donde tiene una alta prevalencia [42]. No obstante, en el análisis multivariado, la desnutrición no se mantuvo como predictor de mortalidad, al igual que en reportes previos $[43,44]$. La hipoalbuminemia ha sido asociada con mortalidad, ventilación mecánica y estancia prolongada en UCIP $[45,46]$, como se observó en este trabajo; sin embargo, no se relacionó con un $A D E$ alto, lo que contrasta con otros datos reportados en adultos $[24,47]$. La determinación de albúmina en nuestro servicio no se realiza sistemáticamente, como sí ocurre con el hemograma al ingreso; la recolección de este dato pudiese no corresponder a la misma muestra de sangre con la que se tomó el $A D E$, además, se solicita en pacientes con sospecha clínica de desnutrición y en aquellos con mayor compromiso clínico, lo que sesga su relación con el ADE.

Las deficiencias de macro y micronutrientes, especialmente el hierro, folatos y vitamina B12 pueden explicar un incremento del $A D E$, sin embargo, Perlstein y colaboradores mostraron que este era un factor independiente asociado a alto riesgo de mortalidad después de ajustar para estas deficiencias [18]. Al igual que en los estudios de Ramby y colaboradores, y Schepens y colaboradores, en este estudio la anemia estaba relacionada con un ADE elevado $[33,37]$. Una de las limitaciones de este estudio es que no se pudieron evaluar todas las posibles variables de confusión, como las medidas de hierro, marcadores del estado nutricional o biomarcadores de inflamación [34]. Un marcador de anemia y desnutrición, como lo es la ferropenia, podría ser evaluado posteriormente, ya que ha sido relacionado con mortalidad en UCIP [48]. 
Respecto a los otros desenlaces, nuestros resultados se asemejan a lo descrito por Schepens y colaboradores, quienes mostraron que estar en el quintil de ADE más alto, se asociaba con mayor necesidad de ventilación mecánica [33]. Estos autores también mostraron que el ADE se correlacionaba inversamente con los días libres de ventilación [33], al igual que el estudio de Said y colaboradores que mostró una relación débil pero significativa [36]. Estos hallazgos validan lo encontrado en nuestra población.

La estancia en UCIP es un indicador de severidad de la enfermedad y calidad de los servicios [49]; nuestra estancia hospitalaria es mayor a la reportada en países desarrollados [50]. Said y colaboradores mostraron una débil asociación entre un valor mayor del ADE y días libres de $\mathrm{UCl}$, en tanto que Ramby y colaboradores informaron que para los pacientes sin sepsis cada aumento del $1 \%$ en el ADE aumentaba 1,17 veces la probabilidad de estancia en UCIP por más de 48 horas $[36,37]$. Sin embargo, en nuestro trabajo no se midieron los días libres de UCIP, y la definición de estancia prolongada no es adecuada para países en vías de desarrollo que tienen condiciones que favorecen una hospitalización más larga y modifiquen la utilidad de los modelos predictivos [51]. A diferencia del estudio de Aslan y colaboradores en adultos con sepsis y sepsis severa, nuestros resultados no muestran una asociación con mayor tiempo de uso de inotrópicos [52].

Debido al diseño transversal de este estudio, no se hizo seguimiento al reingreso de los niños que no fallecieron. Zurauskaite y colaboradores demostraron que un ADE alto se relacionaba con reingresos hospitalarios no planificados en los sobrevivientes [53], lo cual debería ser tenido en cuenta en próxi- mos trabajos como una medida del impacto mediato de la estancia en UCIP.

\section{Conclusión}

Se observó que el ADE al ingreso tiene una asociación moderada con el riesgo de mortalidad en pacientes pediátricos durante la estancia en UCIP, en el contexto de un país en vías de desarrollo. A pesar de que no demostró ser mejor que el PIM2 para pronosticar mortalidad, el hecho de que es un biomarcador asequible y de bajo costo, hace pensar que tal vez su uso en conjunto con PIM2 o con otros biomarcadores [37], aumentaría su capacidad predictiva en la mortalidad de los niños en cuidados intensivos. Se requieren más estudios que evalúen esta posibilidad en nuestro medio.

\section{Agradecimientos}

Los autores agradecen al Pediatra Intensivista Pablo Vásquez Hoyos por su valiosa asesoría.

\section{Confidencialidad de los datos}

No se revelan datos que identifiquen a los pacientes.

\section{Referencias}

1. Bazick HS, Chang D, Mahadevappa K, Gibbons FK, Christopher KB. Red cell distribution width and all-cause mortality in critically ill patients. Crit Care Med 2011;39:1913-1921. https://doi.org/10.1097/ CCM.0b013e31821b85c6.

2. Riley C, Poss WB, Wheeler DS. The evolving model of pediatric critical care delivery in North America. Pediatr Clin North Am 2013;60:545-562. https://doi.org/10.1016/j. pcl.2013.02.001. 
3. Heneghan JA, Pollack MM. Morbidity: Changing the outcome paradigm for pediatric critical care. Pediatr Clin North Am 2017;64:1147-1165. https://doi.org/10.1016/j.pcl.2017.06.011.

4. Arias-Lopez MP, Fernández AL, Ratto ME, Saligari L, Serrate AS, Ko IJ, et al. Pediatric Index of Mortality 2 as a predictor of death risk in children admitted to pediatric intensive care units in Latin America: A prospective, multicenter study. J Crit Care 2015;30:1324-1330. https://doi. org/10.1016/j.jcrc.2015.08.001.

5. Rojas M DV, Ramos J, Molano M. Predictores de mortalidad en la unidad de cuidados intensivos pediátricos del Hospital Universitario de Neiva. 2014. Acta Colomb de Cuid Intensivo 2016;16:1-7. https://doi.org/10.1016/j. acci.2015.10.001.

6. Gulla KM, Sachdev A. Illness severity and organ dysfunction scoring in pediatric intensive care unit. Indian J Crit Care Med 2016;20:27-35. https://doi.org/10.4103/0972-5229.173685.

7. Lee OJ, Jung M, Kim M, Yang HK, Cho J. Validation of the Pediatric Index of Mortality 3 in a Single Pediatric Intensive Care Unit in Korea. J Korean Med Sci 2017;32:365-370. https://doi. org/10.3346/jkms.2017.32.2.365.

8. Slater A, Shann F. The suitability of the Pediatric Index of Mortality (PIM), PIM2, the Pediatric Risk of Mortality (PRISM), and PRISM III for monitoring the quality of pediatric intensive care in Australia and New Zealand. Pediatr Crit Care Med 2004;5:447-454. https://doi. org/10.1097/01.Pcc.0000138557.31831.65.

9. Vincent JL, Opal SM, Marshall JC. Ten reasons why we should NOT use severity scores as entry criteria for clinical trials or in our treatment decisions. Crit Care Med 2010;38:283-287. https:// doi.org/10.1097/CCM.0b013e3181b785a2.

10. Wong HR, Salisbury $S, X i a o ~ Q$, Cvijanovich NZ, Hall M, Allen GL, et al. The pediatric sepsis biomarker risk model. Crit Care 2012;16:R174. https://doi.org/10.1186/cc11652.

11. Wong HR, Cvijanovich NZ, Anas N, Allen GL, Thomas NJ, Bigham MT, et al. Pediatric Sepsis Biomarker Risk Model-II: Redefining the pediatric sepsis biomarker risk model with septic shock phenotype. Crit Care Med 2016;44:2010-2017. https://doi.org/10.1097/ ccm.0000000000001852.
12. Wong HR, Weiss SL, Giuliano JS, Wainwright MS, Cvijanovich NZ, Thomas NJ, et al. Testing the prognostic accuracy of the updated pediatric sepsis biomarker risk model. PLoS One 2014;9:e86242. https://doi.org/10.1371/ journal.pone.0086242.

13. Liu J, Bai C, Li B, Shan A, Shi F, Yao C, et al. Mortality prediction using a novel combination of biomarkers in the first day of sepsis in intensive care units. Sci Rep 2021;11:1275. https:// doi.org/10.1038/s41598-020-79843-5.

14. Constantino BT. Red cell distribution width, revisited. Lab Med 2013;44:e2-e9. https://doi. org/10.1309/lmz1gky9lqtvfbl7.

15. Parker RI. RBC distribution width: Old dog, new trick? Pediatr Crit Care Med 2017;18:193-194. https://doi.org/10.1097/ pcc.0000000000001033.

16. Allen LA, Felker GM, Mehra MR, Chiong JR, Dunlap SH, Ghali JK, et al. Validation and potential mechanisms of red cell distribution width as a prognostic marker in heart failure. J Card Fail 2010;16:230-238. https://doi. org/10.1016/j.cardfail.2009.11.003.

17. Ani C, Ovbiagele B. Elevated red blood cell distribution width predicts mortality in persons with known stroke. J Neurol Sci 2009;277:103108. https://doi.org/10.1016/j.jns.2008.10.024.

18. Perlstein TS, Weuve J, Pfeffer MA, Beckman JA. Red blood cell distribution width and mortality risk in a community-based prospective cohort. Arch Intern Med 2009;169:588-594. https://doi. org/10.1001/archinternmed.2009.55.

19. Zhang $L$, $\mathbf{Y u} \mathbf{C H}$, Guo KP, Huang $\mathrm{CZ}$, Mo LY. Prognostic role of red blood cell distribution width in patients with sepsis: a systematic review and meta-analysis. BMC Immunol 2020;21:40. https://doi.org/10.1186/s12865020-00369-6.

20. Krishna V, Pillai G, Velickakathu-Sukumaran S. Red cell distribution width as a predictor of mortality in patients with sepsis. Cureus 2021;13:e12912. https://doi.org/10.7759/cureus. 12912.

21. Ghimire R, Shakya YM, Shrestha TM, Neupane RP. The utility of red cell distribution width to predict mortality of septic patients in a tertiary hospital of Nepal. BMC Emerg Med 2020;20:43. https://doi.org/10.1186/s12873-020-00337-8. 
22. Bateman RM, Sharpe MD, Singer M, Ellis CG. The effect of sepsis on the erythrocyte. Int J Mol Sci 2017;18:1932. https://doi.org/10.3390/ ijms18091932.

23. Sadaka F, O'Brien J, Prakash S. Red cell distribution width and outcome in patients with septic shock. J Intensive Care Med 2013;28:307-313. https://doi.org/10.1177/0885066612452838.

24. Kim CH, Park JT, Kim EJ, Han JH, Han JS, Choi JY, et al. An increase in red blood cell distribution width from baseline predicts mortality in patients with severe sepsis or septic shock. Crit Care 2013;17:R282. https://doi.org/10.1186/ cc13145.

25. Henry BM, Benoit JL, Benoit S, Pulvino C, Berger BA, Olivera MHS, et al. Red blood cell distribution width (RDW) predicts COVID-19 severity: A prospective, observational study from the Cincinnati SARS-CoV-2 emergency department cohort. Diagnostics (Basel) 2020;10:618. https://doi.org/10.3390/diagnostics10090618.

26. Wang $C$, Zhang $H$, Cao $X$, Deng $R$, Ye Y, Fu Z, et al. Red cell distribution width (RDW): a prognostic indicator of severe COVID-19. Ann Transl Med 2020;8:1230. https://doi.org/10.21037/ atm-20-6090.

27. Lee JJ, Montazerin SM, Jamil A, Jamil U, Marszalek J, Chuang ML, et al. Association between red blood cell distribution width and mortality and severity among patients with $\mathrm{CO}$ VID-19: A systematic review and meta-analysis. J Med Virol 2021;93:2513-2522. https://doi. org/10.1002/jmv.26797.

28. Zinellu A, Mangoni AA. Red blood cell distribution width, disease severity, and mortality in hospitalized patients with sars-cov-2 infection: A systematic review and meta-analysis. J Clin Med 2021;10:286. https://doi.org/10.3390/ jcm10020286.

29. Pouladzadeh M, Safdarian M, Choghakabodi PM, Amini F, Sokooti A. Validation of red cell distribution width as a COVID-19 severity screening tool. Future Sci OA 2021:FSO712. https:// doi.org/10.2144/fsoa-2020-0199.

30. Foy BH, Carlson JCT, Reinertsen E, Padros IVR, Pallares Lopez R, Palanques-Tost E, et al. Association of red blood cell distribution width with mortality risk in hospitalized adults with SARS-CoV-2 infection. JAMA
Netw Open 2020;3:e2022058. https://doi. org/10.1001/jamanetworkopen.2020.22058.

31. Abdelaleem NA, Makhlouf HA, Nagiub EM, Bayoumi HA. Prognostic biomarkers in predicting mortality in respiratory patients with ventilator-associated pneumonia. Egypt J Bronchol 2021;15:16. https://doi. org/10.1186/s43168-021-00062-1.

32. Hashemi SM, Khanbabaee G, Salarian S, Fariborzi M, Kiumarsi A. Association between red cell distribution width and mortality in pediatric patients admitted to intensive care units. Iran J Blood Cancer 2017;9:54-58.

33. Schepens T, De Dooy JJ, Verbrugghe W, Jorens PG. Red cell distribution width (RDW) as a biomarker for respiratory failure in a pediatric ICU. J Inflamm (Lond) 2017;14:12. https://doi.org/10.1186/s12950-017-0160-9.

34. Kim DH, Ha EJ, Park SJ, Jhang WK. Evaluation of the usefulness of red blood cell distribution width in critically ill pediatric patients. Medicine 2020;99:e22075. https:// doi.org/10.1097/MD.0000000000022075.

35. Sachdev A, Simalti A, Kumar A, Gupta N, Gupta D, Chugh P. Outcome prediction value of red cell distribution width in criticallyill children. Indian Pediatr 2018;55:414-416.

36. Said AS, Spinella PC, Hartman ME, Steffen $\mathbf{K M}$, Jackups $\mathbf{R}$, Holubkov $\mathbf{R}$, et al. RBC distribution width: Biomarker for red cell dysfunction and critical illness outcome? Pediatr Crit Care Med 2017;18:134-142. https://doi. org/10.1097/PCC.0000000000001017.

37. Ramby AL, Goodman DM, Wald EL, Weiss SL. Red blood cell distribution width as a pragmatic marker for outcome in pediatric critical illness. PLoS One 2015;10:e0129258. https:// doi.org/10.1371/journal.pone.0129258.

38. Ellahony DM, El-Mekkawy MS, Farag MM. A study of red cell distribution width in neonatal sepsis. Pediatr Emerg Care 2020;36:378-383. https://doi.org/10.1097/ pec.0000000000001319.

39. Guo BF, Sun SZ. Diagnostic accuracy of a dynamically increased red blood cell distribution width in very low birth weight infants with serious bacterial infection. Ital J Pediatr 2021;47:44. https://doi.org/10.1186/s13052 021-00994-w. 
40. Bulut O, Akcakaya A, Bulut N, Ovali F. Elevated red cell distribution width as a useful marker in neonatal sepsis. J Pediatr Hematol Oncol 2021;43:180-185. https://doi. org/10.1097/mph.0000000000002070.

41. Wang F, Pan W, Pan S, Ge J, Wang S, Chen M. Red cell distribution width as a novel predictor of mortality in ICU patients. Ann Med 2011;43:40-46. https://doi.org/10.3109/07853 890.2010 .521766 .

42. Ministerio de Salud y la Protección Social, Instituto Colombiano de Bienestar Familiar y colaboradores. Encuesta Nacional de la Situación Nutricional en Colombia 2015. ENSIN. Bogotá D.C.: Ministerio de salud y la Protección Social; 2015. Acceso 15 de marzo de 2021. Disponible en https://www.icbf.gov. co/sites/default/files/infografia_situacion_ nutricional_0_a_4_anos.pdf.

43. Hulst J, Joosten $K$, Zimmermann L, Hop W, van Buuren S, Büller H, et al. Malnutrition in critically ill children: from admission to 6 months after discharge. Clin Nutr 2004;23:223-232. https://doi. org/10.1016/s0261-5614(03)00130-4.

44. de Souza Menezes F, Leite HP, Koch Nogueira PC. Malnutrition as an independent predictor of clinical outcome in critically ill children. Nutrition 2012;28:267-270. https://doi. org/10.1016/j.nut.2011.05.015.

45. Leite HP, Rodrigues da Silva AV, de Oliveira Iglesias SB, Koch Nogueira PC. Serum albumin is an independent predictor of clinical outcomes in critically ill children. Pediatr Crit Care Med 2016;17:e50-57. https://doi. org/10.1097/pcc.0000000000000596.

46. Durward A, Mayer A, Skellett S, Taylor D, Hanna S, Tibby SM, et al. Hypoalbuminaemia in critically ill children: incidence, prognosis, and influence on the anion gap. Arch Dis Child 2003;88:419-422. https://doi.org/10.1136/ adc.88.5.419.
47. Förhécz Z, Gombos T, Borgulya G, Pozsonyi Z, Prohászka Z, Jánoskuti L. Red cell distribution width in heart failure: prediction of clinical events and relationship with markers of ineffective erythropoiesis, inflammation, renal function, and nutritional state. Am Heart J 2009;158:659-666. https://doi.org/10.1016/j. ahj.2009.07.024.

48. Ong C, Han WM, Wong JJ, Lee JH. Nutrition biomarkers and clinical outcomes in critically ill children: A critical appraisal of the literature. Clin Nutr 2014;33:191-197. https://doi. org/10.1016/j.clnu.2013.12.010.

49. Breslow MJ, Badawi O. Severity scoring in the critically ill: part 2: maximizing value from outcome prediction scoring systems. Chest 2012;141:518-527. https://doi.org/10.1378/ chest.11-0331.

50. Pollack MM, Holubkov $R$, Reeder $R$, Dean JM, Meert KL, Berg RA, et al. PICU length of stay: Factors associated with bed utilization and development of a benchmarking model. Pediatr Crit Care Med 2018;19:196-203. https://doi. org/10.1097/pcc.0000000000001425.

51. Kramer AA, Zimmerman JE. The relationship between hospital and intensive care unit length of stay. Crit Care Med 2011;39:1015-1022. https://doi.org/10.1097/ CCM.0b013e31820eabab.

52. Shaikh MA, Yadavalli DR. Red cell distribution width as a prognostic marker in severe sepsis and septic shock. Int J Adv Med 2017;4:5. https://doi.org/10.18203/2349-3933. ijam20172266.

53. Zurauskaite G, Meier M, Voegeli A, Koch D, Haubitz S, Kutz A, et al. Biological pathways underlying the association of red cell distribution width and adverse clinical outcome: Results of a prospective cohort study. PLoS One 2018;13:e0191280. https://doi.org/10.1371/ journal.pone.0191280. 


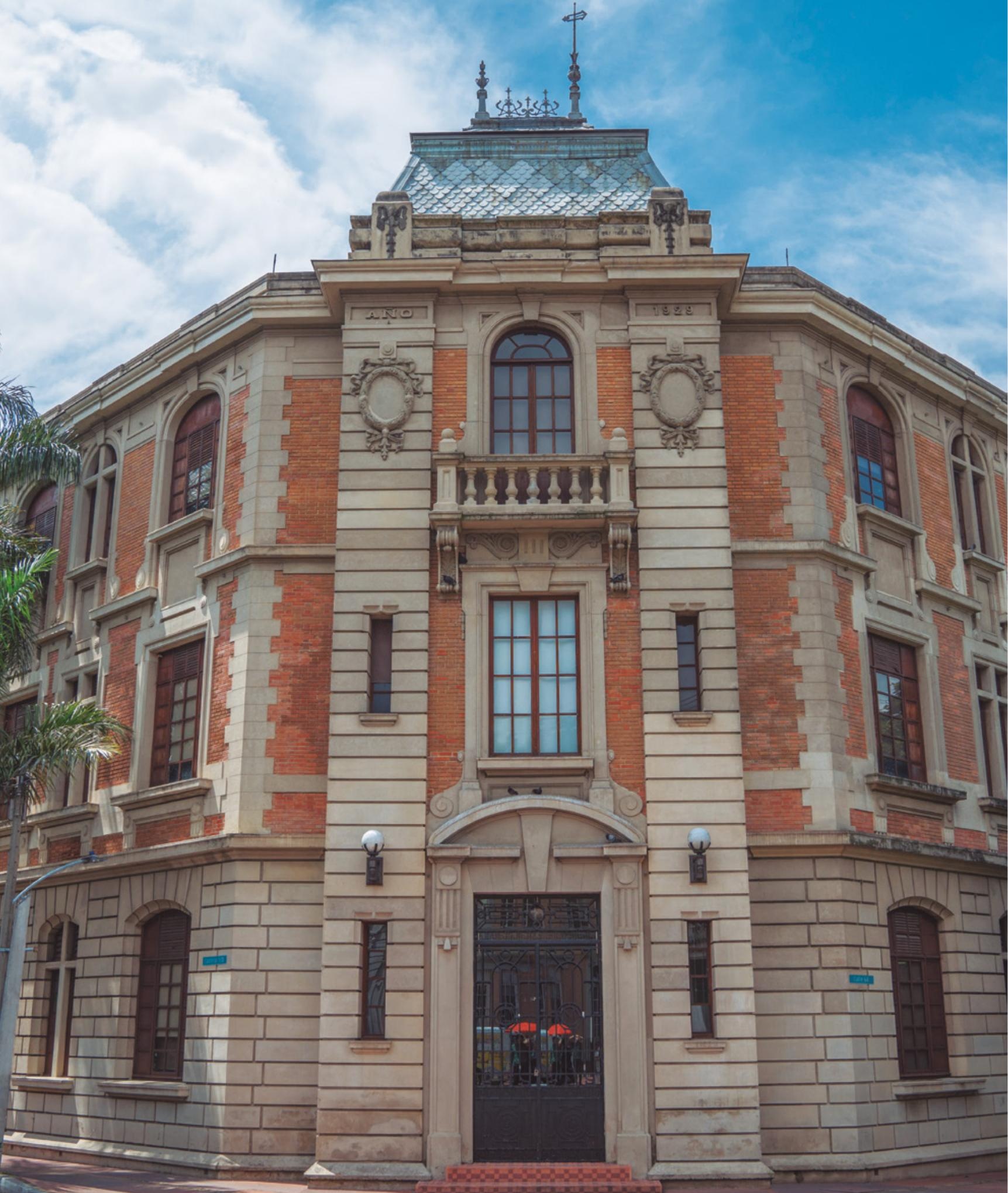

\section{Medicina \& ML Laboralorio.} Educación Médica Continua Certificada

Editora Médica Colombiana S.A., EDIMECO S.A. Carrera 43C No. 5-33 / Teléfono (4)444 4900 Medellín, Colombia 050022
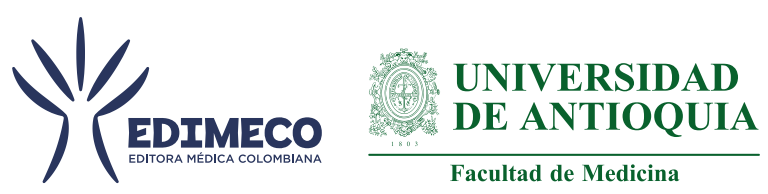

Línea de atención al cliente 018000-941080 phiguita@edimeco.com www.edimeco.com 